\title{
Application of the 3'-Untranslated Region of Messenger RNA from Measles Virus Matrix Protein as an RNA Stabilizer: Implications in Pharmaceutical Biotechnology
}

\author{
Marzieh Marzbany ${ }^{1}$, Fatemeh Ghassemi ${ }^{2}$, \& Mahsa Rasekhian ${ }^{3}$ \\ ${ }^{1}$ Pharmacognosy and Pharmaceutical Biotechnology Group, Kermanshah University of Medical Sciences, \\ School of Pharmacy, Kermanshah, Iran \\ ${ }^{2}$ Virology Department, Pasteur Institute of Iran, Tehran, Iran \\ ${ }^{3}$ Pharmaceutical Sciences Research Center, School of Pharmacy, Kermanshah University of Medical Sciences, \\ Kermanshah, Iran
}

Correspondence: Mahsa Rasekhian, Pharmaceutical Sciences Research Center, School of Pharmacy, Kermanshah University of Medical Sciences, Daneshgah Street, Kermanshah 6734667149, Iran. Tel: +98-83-3427-6489 ext. 304. Fax: +98-83-3427-6493. E-mail: mahsarasekhian@gmail.com

Received: April 20, 2019

doi:10.5539/jmbr.v9n1p53
Accepted: May 9, 2019

Online Published: May 29, 2019

URL: https://doi.org/10.5539/jmbr.v9n1p53

\begin{abstract}
BACKGROUND: The market for the use of recombinant proteins for medical applications has been increasing in recent years. In many cases including fast production of significant amounts of protein for research purposes, transient transfection is the method of choice. In this regard expression vectors are one of the decisive factors in the cost-effectiveness of the production process. The genetic elements found in the 3'untranslated region (UTR) of mRNA expressed by such vectors, play an essential role in determining its stability and thus in the efficiency of the process.
\end{abstract}

METHODS: In this study, the 3'UTR of matrix protein from the Measles Virus (MV) was used to construct a reporter plasmid containing Enhanced Green Fleurocent Protein (EGFP). The reporter construct was transfected into three cell lines. The effect of 3'UTR on mRNA stability was evaluated by real-time PCR. Secondary structure of the mrna was predicted based on minimum free energy. 3'UTR was analyzed in silico for the presence of binding motifs for trans-acting elements with known effects on RNA stability.

RESULTS: Addition of 3'UTR of MV matrix protein sequence to the 3' end of the mRNA, increased the EGFPmRNA stability in time and cell-dependent manner. Analysis for the presence of known cis-acting motifs in 3'UTR indicated the presence of two PABPC1 binding sites, an RNA-binding protein, known for its stability and translation enhancing effects.

CONCLUSION: Our results verified the potential of the 3'UTR region of matrix protein mRNA for improvement of transient recombinant protein production and vector design for mammalian cell hosts.

Keywords: 3'UTR, PABPC1, RNA Stability, Recombinant Protein, Transient Transfection

\section{Background}

The Earth's population is expected to exceed well over 9 billion by 2050, and there is an urgent need to increase the supply of protein from sustainable sources (Ulanova \& Kravchenko, 2016). In recent years, the use of recombinant proteins in the treatment, diagnosis, and prevention of human diseases such as cancer has grown dramatically (Hung et al., 2010; Mahanty, Mishra, Bosu, Maurya, Netam, \& Sarkar, 2013; Lagasse et al., 2017).

The achievement of high cellular production efficiency is the essential factor in determining the cost-effectiveness of the recombinant protein production process, even for high-value proteins such as monoclonal antibodies. High efficiency becomes even more critical when patents expire. So far, many efforts have been made to increase the effectiveness of the production of recombinant proteins by altering the various process steps (Gustafsson, Minshull, Govindarajan, Ness, Villalobos, \& Welch, 2012).

The stream of information from a heterologous gene to a secreted pharmaceutical protein comprises of several steps including transcription, mRNA processing, and transport and translation, each of which contributes to the 
yield of the protein production process.

Commercial expression vectors are often assemblies of natural genetic elements such as promoters and terminators chosen from a constrained source of available control components. Such vectors are known to be a critical deciding variable in the proficiency of protein production. Owing to the compiling research to find new elements, the number of potentially useful vector elements is expanding (Kado, 2014; Kaufman, 2000). The utilization of strong promoters and the design and configuration of the plasmid are examples of methodologies that have been inspected before and appeared to enhance the profits (Hung et al., 2010; Chen, Li, \& Tian, 2016).

In eukaryotic cells, RNA surveillance machinery supervises the quality and the abundance of mRNAs. Messenger RNA degradation, as a critical component of RNA surveillance machinery, plays a pivotal role in the regulation of gene expression.

In this regard, browsing the genetic elements of RNA viruses can provide a good source for innovation in the design of RNA molecules with modified stability and half-life. Mainly, because they effectively interact with cellular RNA surveillance machinery to achieve an ideal balance between viral and cellular protein expression. Making such a balance is vital to building up contamination in the host cell (Dickson \& Wilusz, 2011; Narayanan \& Makino, 2013).

Thereby, 5' and 3'untranslated (UTR) region in viral and eukaryotic mRNA contains regulatory elements. These regulatory elements play an essential role in the fate of mRNA through interaction with cellular factors. While 5'UTR has a more critical role in the start of translation, 3'UTR oversees numerous aspects of post-transcriptional gene regulation. This region is variable in both length and sequence; it locates between the stop codon and the poly (A) tail (Schwerk \& Savan, 2015).

Measles Virus, an enveloped virus abbreviated as MV, falls in genus Morbillivirus from Paramyxoviridae family. It contains a non-segmented RNA genome of negative sensitivity which codes for six genes (Noton \& Fearns, 2015). They are hemagglutinin (H), fusion (F), large (L) proteins, matrix (M) and phosphor (P). The M protein is essential in virus assembly and also modulating the synthesis of viral RNA (Iwasaki, Takeda, Shirogane, Nakatsu, Nakamura, \& Yanagi, 2009; Hirano, Wang, Gombart, \& Wong, 1992; Riedl, Moll, Klenk, \& Maisner, 2002; Yu, Shahriari, Li, \& Ghildyal, 2016). Regrettably, the literature contains only a few studies on the effect of the 3'UTR genetic elements of RNA viruses on increasing the transcript stability and translation efficiency. In the present study, we investigated the effect of 3'UTR of matrix protein mRNA from MV on the stability of mRNA of EGFP (enhanced Green fluorescent protein). Our findings show that application of 3'UTR of matrix protein mRNA from MV affects the stability of EGFP mRNA positively albeit in a cell line and time-dependent manner. We believe that the application of such elements offers substantial potential in the design and construction of a new generation of expression vectors with the aim of increased benefits in the production process of recombinant therapeutic proteins.

\section{Methods}

\subsection{Selection of Sequence Data Set}

MV strains with a complete genome sequence were extracted from HTTP:// www.ncbi.nlm.nih.gov website for National Centre for Biotechnology Information.

Only sequences with a total length of $15894 \mathrm{nt}$ were retrieved for further analysis. Total of 64 complete genomes was selected. Selected sequences were cross-referenced against Reference strains addressed by the Center for Disease Control and Prevention at https://www.cdc.gov/measles/lab-tools/who-table.html. Alignment was created by CLC Main Workbench software (versionb5.5). A 427 length consensus nucleotide sequence (immediately after the TAG stop codon of M gene mRNA) was chosen as the 3'UTR of the M gene.

The chosen consensus sequence had more than 95\% homology with the 3'UTR sequence of the M gene in AB012948 strain, hence nucleotide sequence 4447- 4871 of AB012948 strain was chosen for chemical synthesis.

\subsection{Plasmid Design and Synthesis}

EGFP sequence was amplified by specific PCR primers (sequence not shown) and pEGFPN-1 plasmid as a template. Amplified sequence was cloned into the EcoRV restriction site of pcDNA(+) TM vector by standard protocols. This plasmid was called PCDNA and was used as a reference sample for calculation of relative expression of EGFP (Rao, Huang, Zhou, \& Lin, 2013). The selected sequence of the 3'UTR of measles virus matrix protein was ordered for chemical synthesis by BIOMATIK (Cambridge, Ontario, Canada). The sequence was primarily cloned in pBSK(+)Simple-Amp by the manufacturer. The sequence was then sub-cloned into PCDNA vector by XhoI and XbaI restriction enzymes. The resulting plasmid was called 3UTR and was used as 
the target sample for calculation of relative expression of EGFP (Figure 1). Accuracy of recombinant plasmids was confirmed by restriction enzyme analysis and sequencing (data not shown).

A

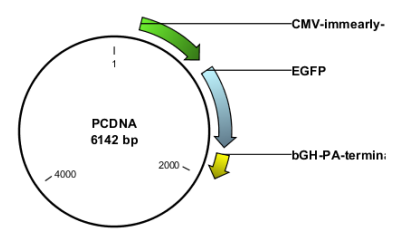

B

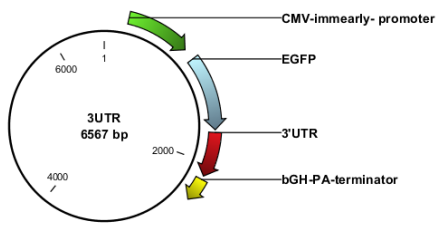

Figure 1. Construction of recombinant plasmid vectors. PCDNA (A) was constructed by EGFP amplification and standard cloning into pcDNA(+) TM vector. 3UTR (B) was constructed by standard cloning of chemically synthesized sequence of 3'UTR of measles virus matrix protein gene at the 3'UTR end of EGFP in recombinant PCDNA

\subsection{Prediction of RNA Secondary Structure}

Prediction of the possible folding of EGFP mRNA transcribed from PCDNA or 3UTR constructs was made based on Minimum Free Energy (MFE) with the online http://rna.tbi.univie.ac.at/cgi-bin/RNAWebSuite/RNAfold.cgi (Gruber, Lorenz, Bernhart, Neuböck, \& Hofacker, 2008). DNA sequences were selected from initiation point of the transcription $(+1 \mathrm{nt})$ of putative CMV promoter of pcDNA3.1 $(+)^{\mathrm{TM}}$ vector to its putative polyA signal including the particular insert sequence. Calculated MFE was subsequently used to obtain the Free energy density indicator (Adjusted minimum free energy (AMFE)), by dividing MFE by the sequence length. The results were then multiplied by 100 to relate the index to a segment of 100 nucleotides (Trotta, 2014). After calculation, AMFEs were 33 for 3UTR and 36 for PCDNA mRNAs.

\subsection{Analysis of Measles Virus Matrix Protein Gene 3'UTR for Known RBP Interactions}

Search for the presence of any previously described motifs (with known RNA stability modulating effects or binding sites for RNA Binding Proteins (RBP) in the 3'UTR sequence of MV matrix protein gene was based on the available data and analysis by RBPDB: The database of RNA-binding specificities (http://rbpdb.ccbr.utoronto.ca/).

\subsection{Cell Lines and Transfection}

BHK, COS-7 , and CHO DG44 cell lines (purchased from the Pasteur Institute of Iran) were cultured. As literature has revealed, several factors including environmental factors and genetic factors also influence the growth of cells in culture (Sathya, Mathialagan, Bai, \& Ramalingam, 2009). In this study, the cells were cultured in DMEM/F12 medium supplemented with $10 \%$ bovine calf serum, $100 \mathrm{U} / \mathrm{ml}$ penicillin and $100 \mu \mathrm{g} / \mathrm{ml}$ streptomycin. Cells were maintained at $37^{\circ} \mathrm{C}$ in a humidified incubator with $5 \% \mathrm{CO}_{2}$. All transfection procedures were carried out by Effectene Transfection Reagent (qiagen ${ }^{\mathrm{TM}}$ ) according to the manufacturer's protocol. Briefly, the day before transfection $1-2 \times 105$ cells were seeded in 24 well cell culture plates to achieve $80 \%$ of confluency at the day of transfection. At the proper confluency, cells were transfected with $200 \mathrm{ng}$ of either PCDNA or 3UTR. All transfection experiments were carried out in triplicate. Transfected cells were maintained at $37^{\circ} \mathrm{C}$ in a humidified incubator supplemented with $5 \% \mathrm{CO}_{2}$.

\subsection{RNA Extraction and EGFP Relative Expression Assay}

One day after transfection, cells were evaluated for EGFP expression by fluorescent microscopy. Then, cells were covered with an overlay of culture media containing $5 \mu \mathrm{g}$ actinomycin D (Sigma-Aldrich) to stop transcription. Subsequently, total RNA was extracted by RNeasy kit (Qiagen) according to the manufacturer protocol at $0,2,4,6,8$ and 10 hour time intervals after actinomycin D treatment. The RNA concentration was assessed at 260, and the ratio of 260/280 OD was used as an indicator for RNA quality. All RNA samples were subjected to DNase I treatment (Thermoscientific) for 45 minutes at $37^{\circ} \mathrm{C}$ To remove any potential DNA contamination. DNase I was further inactivated by heat $\left(65^{\circ} \mathrm{C}\right.$ for 15 minutes). All real-time PCR analyses were carried out using a Rotor Gene 6000 system (Corbett Research, Australia) and sequence-specific primers for EGFP and GAPDH (Table 1). In brief, 20ul reactions were set up using Power SYBR ${ }^{\circledR}$ Green RNA-to-CT ${ }^{\mathrm{TM}}$ 1-Step Kit (Thermoscientific), and ten ng of the extracted RNA was used as a template. Samples were normalized to the control housekeeping gene "GAPDH." Relative expression levels were calculated using the $2^{-(\Delta \Delta(\mathrm{CT}))}$ method (Livak \& Schmittgen, 2001). All Real Time analyses were carried out in triplicate. 
Table 1. Name and sequence of primers used in evaluation of relative expression of EGFP by RT-PCR

\begin{tabular}{ll}
\hline primer name & $5^{\prime}$ sequence $3^{\prime}$ \\
\hline EGFP forward & ctg ctg ccc gac aac ca \\
EGFP revers & acc atg tga tcg cgc ttc tc \\
GAPDH forward & Tcc tgc acc acc aac tgc tta \\
GAPDH revers & Tcc aca gtc ttc tgg gtg gca \\
\hline
\end{tabular}

\section{Statistical Analysis}

The analysis of the results was computed using the SPSS package. It was mostly computation of the mean, factorial analysis, and the standard deviation. All values with $\mathrm{P}$ value less than 0.05 were considered to be significant.

\section{Results}

\subsection{Secondary Structure Prediction Showed a More Stable Ensemble for 3UTR}

Thermodynamic analyses based on MFE calculations for optimum secondary structures of 3UTR and PCDNA mRNA (Figure 2) indicated $\Delta \mathrm{G}$ values of $-461.81 \mathrm{kcal} / \mathrm{mol}$ and $-359.75 \mathrm{kcal} / \mathrm{mol} \mathrm{MFE} \mathrm{respectively.} \mathrm{To} \mathrm{normalize}$ the predicted $\Delta \mathrm{G}$ to the length of mRNA, $\Delta \mathrm{G}$ was divided by the number of nucleotides in the sequence; 1404 and 979 for 3UTR and PCDNA, respectively. Results showed that free energy density indicators equal to 33 and 36 for 3UTR and PCDNA apiece (Lorenz et al., 2011; Freyhult, Gardner, \& Moulton, 2005). Results showed that free energy density indicators equal to 33 and 36 for 3 UTR and PCDNA respectively.
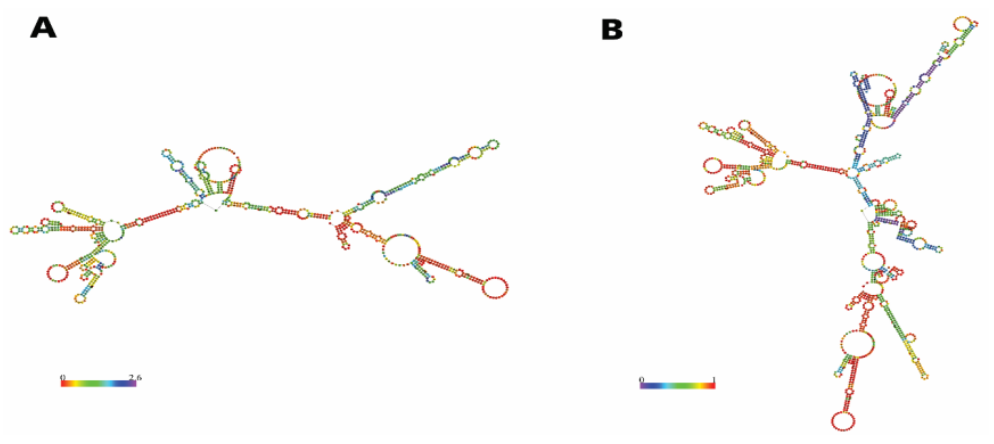

Figure 2. Prediction of RNA secondary structure was based on MFE. RNA fold web server was used in secondary structure prediction of sequences from constructs of PCDNA (A) and 3UTR (B)

(http://rna.tbi.univie.ac.at/cgibin/ RNAfold.cgi). From the AMFE prediction, PCDNA (36) has a slightly more stable secondary structure

\subsection{3'UTR of Measles Virus Matrix Protein mRNA is a Target for Human RBP}

Analysis of the 3'UTR sequence of measles virus (strain AB012948, nt 4447 to 4871) matrix protein mRNA for known human RNA binding proteins in RBPDB showed that based on position weight matrices (PWMs) PAPBC1 (cytoplasmic polyadenylate-binding protein) is the top hit for interaction with our RNA sequence. Relative score (the percent of score obtained corresponding to the possible maximum PWM score) for PAPBC1 communication was $100 \%$ (Table 2).

Table 2. Score and matching sequence of RBPs with possible interaction with 3'UTR sequence of measles virus (strain AB012948, nt 4447 to 4871) matrix protein mRNA. (http://rbpdb.ccbr.utoronto.ca)

\begin{tabular}{llllll}
\hline Score & Relative score & RBP name & start & end & Matching sequence \\
\hline 8.7178165 & $100 \%$ & PABPC1 & 63 & 67 & AAAAA \\
8.7178165 & $100 \%$ & PABPC1 & 64 & 68 & AAAAA \\
\hline
\end{tabular}

\subsection{3'UTR of Measles Virus Matrix Protein mRNA Enhances the Expression of EGFP in All Three Cell Lines}

As shown in Figure 3, placement of the 3'UTR sequence of MV Matrix protein mRNA downstream of the EGFP coding sequence, affected the level of mRNA in all cell lines compared to the control construct. 


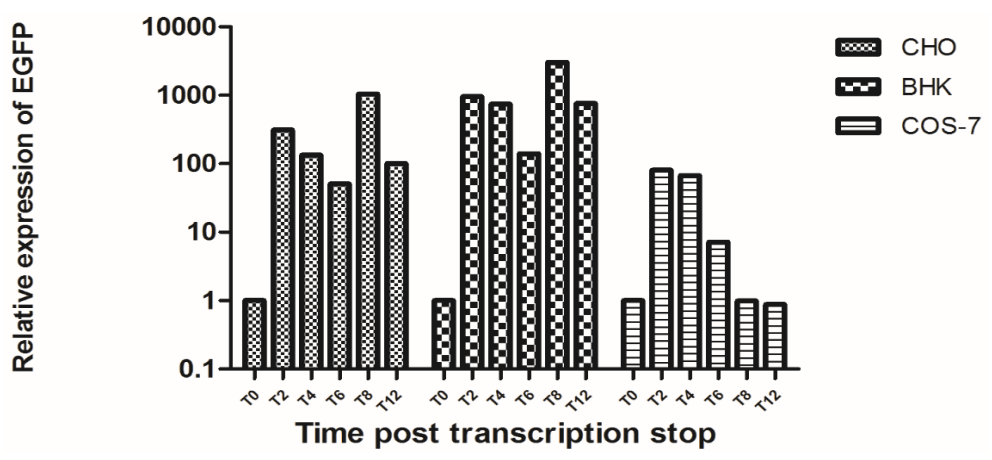

Figure 3. Relative transcription levels of PCDNA versus 3UTR. After treatment with Actinomycin D, RNA was extracted form cells during $0,2 \mathrm{nd}, 4$ th, 6 th, 8 th and 12 th hours. $2^{-(\Delta \Delta(\mathrm{CT}))}$ was used to calculate Relative transcription rates $(\mathrm{n}=3)$

In $\mathrm{CHO}$ and BHK cell lines, the level of 3UTR mRNA was always higher than that of PCDNA with a pick at T8 but in the case of COS-7, 3utr mRNA levels started to decrease after T4, and in the following time points mRNA from both constructs were equally abundant in COS-7. Factorial analysis of variance demonstrated a significant effect for cell line $(\mathrm{P}<0.0001)$, and time interval $(\mathrm{P}<0.0001)$. Analyses of the interactions between cell line and time interval proved that all measures were statistically significant $(\mathrm{P}<0.0001)$. Therefore, according to the obtained results and statistical analyses, application of the 3'UTR of MV Matrix protein mRNA regulated the EGFP mRNA levels in a time and cell line-dependent manner.

\section{Discussion}

In the past decade, Recombinant therapeutic proteins have changed the approach of modern medicine toward previously refractory diseases and diagnostic procedures (Jayapal, Wlaschin, Hu, \& Yap, 2007). Cultivated mammalian cells such as BHK and $\mathrm{CHO}$ have become the dominant system for the production of recombinant proteins for clinical applications because of their capacity for proper protein folding, assembly and post-translational modification (Wurm, 2004; Estes \& Melville, 2014). Transient gene expression (TGE) is an accelerated approach for recombinant protein production compared to the time- and labor-consuming step of creating stable cell lines. Using this approach cells are directly cultured (and transfected) at the production (Bandaranayake \& Almo, 2014; Backliwal, Hildinger, Chenuet, Wulhfard, De Jesus, \& Wurm, 2008). The yield of protein expression by TGE vectors depends on multiple factors including mRNA stability (Kaufman, 2000). Many of the factors that decide the stability and fate of the mRNA molecule are located at the 3'UTR of the targeted transcript (Schwerk \& Savan, 2015; Schwanhausser et al., 2011; Morris, Mukherjee, \& Keene, 2010; Lopez de Silanes, Quesada, \& Esteller, 2007). Since viruses must employ fundamental defense mechanisms to protect their genetic information from strict surveillance machinery of eukaryotic cells, the untranslated region of viral genomes is a credible source to explore for such elements. In the present study, we for the first time evaluated the effect of the 3'UTR sequence from matrix protein mRNA of MV, on mRNA stability for potential biotechnological applications in the manufacturing of the therapeutic proteins in three mammalian cell lines.

Our results showed that despite a slightly more stable ensemble for PCDNA; which was predicted base on AMFE, the abundance of 3UTR mRNA was higher than that of PCDNA in all three cell lines at all times (Figure $3)$. This finding is in line with the results of previous research that showed MFE alone is not a suitable measure for determining the RNA stability ( $\mathrm{Jia} \& \mathrm{Li}, 2005$ ). It has previously been proven that one of the most critical determinants of RNA stability is the interaction of RNA with RNA binding proteins (RBP) (Bolognani \& Perrone-Bizzozero, 2008). Evaluation of the 3UTR mRNA showed that this sequence is a possible target for PABPC1 (Table 2). Research indicates that PABPC1, interact not only with the poly(A) tail, but also with specific sequences in the mRNA, and they have a particular role on the metabolism of mRNAs (Goss \& Kleiman, 2013; Mangus, Evans, \& Jacobson, 2003; Le et al., 1997).

Viral infections are considered as one of the principal threats to human life and health worldwide (Mohamed, Zohny, El-Senousy, \& Abou El-Elaa, 2018). Findings of Mckinney et al. is in line with our results, they have shown that following the human cytomegalovirus infection, an increase in the concentration of PABPC1 as a key translational factor, is a crucial step in establishing an infection (Smith et al., 2017; McKinney, Yu, \& Mohr, 2013).

Previous publications on interactions of influenza virus, NS1 protein and PABPC1 showed that PABP1, NS1, 
mRNAs and eIF4GI interaction led to enhanced RNA translation through a specific ribosome complex recruitment (Burgui, Aragon, Ortin, \& Nieto, 2003).

As stated in the results, Analysis of variance showed that 3UTR mRNA stability was increased in a time and cell line-dependent manner. Such a pattern was reported in a previous work by Azadmanesh et al. (Rasekhian, Roohvand, Teimoori-Toolabi, Amini, \& Azadmanesh, 2014). In all three cell lines, the abundance of 3UTR mRNA was always higher than PCDNA mRNA, and the most significant difference in the relative abundance of mRNA copy numbers was observed in T2. Since actinomycin D stopped the translation, it seems that a sudden decrease in the copy number of the less stable PCDNA mRNA has contributed to this observation. In BHK and CHO, the difference in 3UTR and PCDNA mRNA copy number decreased in the following time points. However, at T8 another substantial difference in relative mRNA abundance was observed. Since the concentrations of actinomycin D used for inhibition of RNA polII activity are much lower than that of actinomycin D IC50 $(0.004 \mathrm{M}$ vs. $0.42 \mathrm{M})$, this phenomenon can be attributed to the removal of RNAPol II containment. In COS-7 however, the relative expression of 3UTR mRNA showed a descending trend which can be contributed to a distinct pattern of expression of cellular translation enhancing factors, including but not limited to PABPC1. We did not further investigate this phenomenon in the present study, and unfortunately, we did not find a piece of appealing evidence in the literature.

Based on our results, we suggest that further investigation on the nature of the interaction between the 3'UTR sequence of mRNA transcribed from the MV matrix protein gene and cellular trans-acting factors is necessary. Additionally, site-directed mutagenesis in the 3'UTR sequence of mRNA transcribed from the MV matrix protein gene with the aim of modified interaction between RNA sequence and RBPs with known RNA-stabilizing effects is of our team's interest.

\section{Conclusion}

We believe viruses especially RNA viruses are yet an unexplored source of genetic elements for biotechnological applications and they can provide a strong potential for designing a new generation of vectors for increased industrial yields in recombinant protein production.

\section{Conflict of interests}

The authors declare that there is no conflict of interests regarding the publication of this paper.

\section{References}

Backliwal, G., Hildinger, M., Chenuet, S., Wulhfard, S., De Jesus, M., \& Wurm, F. M. (2008). Rational vector design and multi-pathway modulation of HEK 293E cells yield recombinant antibody titers exceeding $1 \mathrm{~g} / \mathrm{l}$ by transient transfection under serum-free conditions. Nucleic Acids Research, 36, e96.

Bandaranayake, A. D., \& Almo, S. C. (2014). Recent advances in mammalian protein production. FEBS Letters, $588,253-260$.

Bolognani, F., \& Perrone-Bizzozero, N. I. (2008). RNA-protein interactions and control of mRNA stability in neurons. Journal of Neuroscience Research, 86, 481-489.

Burgui, I., Aragon, T., Ortin, J., \& Nieto, A. (2003). PABP1 and eIF4GI associate with influenza virus NS1 protein in viral mRNA translation initiation complexes. The Journal of General Virology, 84, 3263-3274.

Chen, L., Li, Y., \& Tian, P. (2016). Enhanced Promoter Activity by Replenishment of Sigma Factor rpoE in Klebsiella pneumoniae. Indian Journal of Microbiology, 56, 190-197.

Dickson, A. M., \& Wilusz, J. (2011). Strategies for viral RNA stability: Live long and prosper. Trends in Genetics: TIG, 27, 286-293.

Estes, S., \& Melville, M. (2014). Mammalian cell line developments in speed and efficiency. Adv Biochem Eng Biotechnol, 139, 11-33.

Freyhult, E., Gardner, P. P., \& Moulton, V. (2005). A comparison of RNA folding measures. BMC Bioinformatics, 6, 241.

Goss, D. J., \& Kleiman, F. E. (2013). Poly(A) binding proteins: Are they all created equal? Wiley Interdisciplinary Reviews. RNA 4, 167-179.

Gruber, A. R., Lorenz, R., Bernhart, S. H., Neuböck, R., \& Hofacker, I. L. (2008). The Vienna RNA Websuite. Nucleic Acids Research, 36, W70-W74. 
Gustafsson, C., Minshull, J., Govindarajan, S., Ness, J., Villalobos, A., \& Welch, M. (2012). Engineering genes for predictable protein expression. Protein Expression and Purification, 83, 37-46.

Hirano, A., Wang, A. H., Gombart, A. F., \& Wong, T. C. (1992). The matrix proteins of neurovirulent subacute sclerosing panencephalitis virus and its acute measles virus progenitor are functionally different. Proceedings of the National Academy of Sciences, 89, 8745-8749.

Hung, F., Deng, L., Ravnikar, P., Condon, R., ..., \& Shi, S. (2010). mRNA stability and antibody production in CHO cells: Improvement through gene optimization. Biotechnology Journal, 5, 393-401.

Iwasaki, M., Takeda, M., Shirogane, Y., Nakatsu, Y., Nakamura, T., \& Yanagi, Y. (2009). The matrix protein of measles virus regulates viral RNA synthesis and assembly by interacting with the nucleocapsid protein. Journal of Virology, 83, 10374-10383.

Jayapal, K. P., Wlaschin, K. F., Hu, W., \& Yap, M. G. (2007). Recombinant protein therapeutics from CHO cells-20 years and counting. Chemical Engineering Progress, 103, 40.

Jia, M., \& Li, Y. (2005). The relationship among gene expression, folding free energy and codon usage bias in Escherichia coli. FEBS Letters, 579, 5333-5337.

Kado, C. I. (2014). Historical Events That Spawned the Field of Plasmid Biology. Microbiology Spectrum, 2.

Kaufman, R. J. (2000). Overview of vector design for mammalian gene expression. Molecular Biotechnology, 16, 151-160.

Lagasse, H. A., Alexaki, A., Simhadri, V. L., Katagiri, N. H., ..., \& C. Kimchi-Sarfaty, C. (2017). Recent advances in (therapeutic protein) drug development. F1000Research, 6, 113.

Le, H., Tanguay, R. L., Balasta, M. L., Wei, C. C., ..., \& Gallie, D. R. (1997). Translation initiation factors eIF-iso4G and eIF-4B interact with the poly(A)-binding protein and increase its RNA binding activity. The Journal of Biological Chemistry, 272, 16247-16255.

Lopez de Silanes, I., Quesada, M. P., \& Esteller, M. (2007). Aberrant regulation of messenger RNA 3'-untranslated region in human cancer. Cellular Oncology: the Official Journal of the International Society for Cellular Oncology, 29, 1-17.

Lorenz, R., Bernhart, S. H., Siederdissen, C. H. Z., Tafer, H., ..., \& Hofacker, I. L. (2011). ViennaRNA Package 2.0. Algorithms for Molecular Biology: AMB, 6, 26.

Mahanty, A., Mishra, S., Bosu, R., Maurya, U., Netam, S. P., \& Sarkar, B. (2013). Phytoextracts-Synthesized Silver Nanoparticles Inhibit Bacterial Fish Pathogen Aeromonas hydrophila. Indian Journal of Microbiology, 53, 438-446.

Mangus, D. A., Evans, M. C., \& Jacobson, A. (2003). Poly(A)-binding proteins: Multifunctional scaffolds for the post-transcriptional control of gene expression. Genome Biology, 4, 223.

McKinney, C., Yu, D., \& Mohr, I. (2013). A new role for the cellular PABP repressor Paip2 as an innate restriction factor capable of limiting productive cytomegalovirus replication. Genes \& Development, 27, 1809-1820.

Mohamed, M. S., Zohny, Y. M., El-Senousy, W. M., \& Abou El-Elaa, A. M. (2018). Synthesis and Biological Screening of Novel Pyrazoles and their Precursors as Potential Antiviral Agents. Pharmacophore, 9(1), 126139.

Morris, A. R., Mukherjee, N., \& Keene, J. D. (2010). Systematic analysis of posttranscriptional gene expression Wiley interdisciplinary reviews. Systems Biology and Medicine, 2, 162-180.

Narayanan, K., \& Makino, S. (2013). Interplay between viruses and host mRNA degradation. Biochimica et Biophysica Acta, 1829, 732-741.

Noton, S. L., \& Fearns, R. (2015). Initiation and regulation of paramyxovirus transcription and replication. Virology, 479, 545-554.

Rao, X., Huang, X., Zhou, Z., \& Lin, X. (2013). An improvement of the $2^{\wedge}$ (-delta delta CT) method for quantitative real-time polymerase chain reaction data analysis. Biostatistics, Bioinformatics and Biomathematics, 3, 71-85.

Rasekhian, M., Roohvand, F., Teimoori-Toolabi, L., Amini, S., \& Azadmanesh, K. (2014). Application of the 3'-noncoding region of poliovirus RNA for cell-based regulation of mRNA stability: Implication for biotechnological applications. Biotechnology and Applied Biochemistry, 61, 699-706. 
Riedl, P., Moll, M., Klenk, H. D., \& Maisner, A. (2002). Measles virus matrix protein is not cotransported with the viral glycoproteins but requires virus infection for efficient surface targeting. Virus Research, 83, 1-12.

Sathya, R., Mathialagan, A., Bai, B. F., \& Ramalingam, K. (2009). Determinant factors for hybridmyeloma culture (H9r9) in the yield of anti - D - impact of medium (IMDM and RPMI), serum (FCS) and cell density. J Biochem Tech, 1(2), 53-56

Schwanhausser, B., Busse, D., Li, N., Dittmar, G., .., \& Selbach, M. (2011). Global quantification of mammalian gene expression control. Nature, 473, 337-342.

Schwerk, J., \& Savan, R. (2015). Translating the Untranslated Region. Journal of Immunology, 195, 2963-2971.

Smith, R. W. P., Anderson, R. C., Larralde, O., Smith, J. W. S., ..., \& Gray, N. K. (2017). Viral and cellular mRNA-specific activators harness PABP and eIF4G to promote translation initiation downstream of cap binding. Proceedings of the National Academy of Sciences of the United States of America, 114, 6310-6315.

Trotta, E. (2014). On the normalization of the minimum free energy of RNAs by sequence length. PloS One, 9 , e113380.

Ulanova, R., \& Kravchenko, I. (2016). Housefly Larvae as a Source of Good Quality Renewable Protein Product. Entomology and Applied Science Letters, 3(5), 182-188.

Wurm, F. M. (2004). Production of recombinant protein therapeutics in cultivated mammalian cells. Nature Biotechnology, 22, 1393-1398.

Yu, X., Shahriari, S., Li, H. M., \& Ghildyal, R. (2016). Measles virus matrix protein inhibits host cell transcription. PloS One, 11, e0161360.

\section{Appendix}

\section{List of abbreviations:}

3'UTR: 3'untranslated region

EGFP: enhanced green fluorescent protein

MV: measles virus

RBP: RNA binding protein

PABPC1: Polyadenylate-binding protein 1

\section{Declarations:}

Ethics approval and consent to participate: This article does not contain any studies with human participants or animals performed by any of the authors. This manuscript has been approved by Ethical committee of KUMS (IR.KUMS.REC.1396.585).

Consent for publication: not applicable

Availability of data and material: not applicable

Competing interests: The authors declare that they have no competing interests

Funding: This study was funded by Kermanshah University of Medical Sciences.

Authors' contributions: M.M has performed all laboratory work. F.G has contributed to development of idea and preparation of the manuscript. M.R has developed the main Idea and prepared the manuscript and has participated in the laboratory work.

\section{Copyrights}

Copyright for this article is retained by the author(s), with first publication rights granted to the journal.

This is an open-access article distributed under the terms and conditions of the Creative Commons Attribution license (http://creativecommons.org/licenses/by/4.0/). 